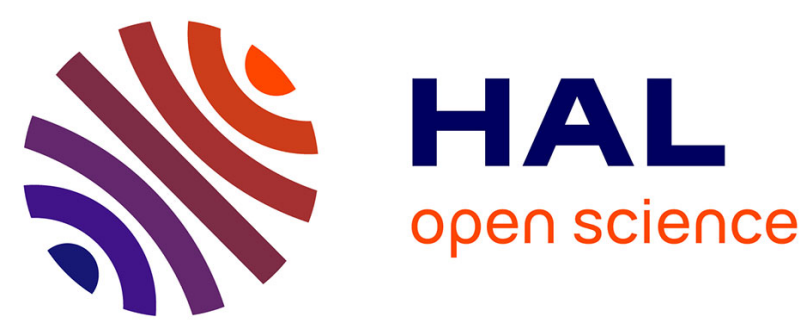

\title{
Design and analysis of a finite volume scheme for a concrete carbonation model
}

Claire Chainais-Hillairet, Antoine Zurek, Benoît Merlet

\section{To cite this version:}

Claire Chainais-Hillairet, Antoine Zurek, Benoît Merlet. Design and analysis of a finite volume scheme for a concrete carbonation model. FVCA8 2017 - International Conference on Finite Volumes for Complex Applications VIII, Jun 2017, Lille, France. pp.285-292, 10.1007/978-3-319-57397-7_21. hal-01645137

\section{HAL Id: hal-01645137 https://hal.science/hal-01645137}

Submitted on 30 Nov 2017

HAL is a multi-disciplinary open access archive for the deposit and dissemination of scientific research documents, whether they are published or not. The documents may come from teaching and research institutions in France or abroad, or from public or private research centers.
L'archive ouverte pluridisciplinaire HAL, est destinée au dépôt et à la diffusion de documents scientifiques de niveau recherche, publiés ou non, émanant des établissements d'enseignement et de recherche français ou étrangers, des laboratoires publics ou privés. 


\title{
Design and analysis of a finite volume scheme for a concrete carbonation model
}

\author{
Claire Chainais-Hillairet, Benoît Merlet and Antoine Zurek
}

\begin{abstract}
In this paper we introduce a finite volume scheme for a concrete carbonation model proposed by Aiki and Muntean in [1]. It consists in a Euler discretisation in time and a Scharfetter-Gummel discretisation in space. We give here some hints for the proof of the convergence of the scheme and show numerical experiments.
\end{abstract}

Key words: Finite volume scheme, convergence analysis, free-boundary system

MSC (2010): 65M08, 65N08, 35Q30

\section{Introduction}

The carbonation phenomenon is a physico-chemical reaction which is the main cause of concrete structure degradation. We can describe the carbonation process as follows: $\mathrm{CO}_{2}(\mathrm{~g})$ from the atmosphere enters in the concrete and it is quickly transformed in $\mathrm{CO}_{2}(\mathrm{aq})$. The introduction of $\mathrm{CO}_{2}(\mathrm{aq})$ in concrete drives the carbonation reaction. This reaction facilitates a drop of the $\mathrm{pH}$ inside the material and allows the corrosion process to damage the metallic reinforcement bars. It deteriorates the concrete and reduces the durability of the structure.

In order to model this phenomenon Aiki and Muntean proposed in [1] a freeboundary system where the varying space domain represents the carbonated zone. The unknowns $u$ and $v$ represent the mass concentration of $\mathrm{CO}_{2}$ respectively in water and air and $s$ represents the penetration depth which measures the size of the

Univ. Lille, CNRS, UMR 8524 - Laboratoire Paul Painlevé,

59000 Lille, France

e-mail: claire.chainais@math.univ-lille1.fr

benoit.merlet@math.univ-lille1.fr

antoine.zurek@inria.fr 
carbonated zone. The unknowns $u$ and $v$ solve a weakly coupled system of reactiondiffusion equations in the varying domain $(0, s(t))$ and $s$ solves an ordinary differential equation. The concentrations $u$ and $v$ are subjected to Dirichlet boundary conditions at $y=0$ and Robin/Neumann boundary conditions at $y=s(t)$.

For this system Aiki and Muntean have shown in [1] the existence and the uniqueness of a global solution. They also have shown that the penetration depth follows a $\sqrt{T}$-law of propagation in $[2,3]$. Our aim is to define a finite volume scheme for the concrete carbonation model and to show the convergence of the numerical scheme. In this short paper, we just give sketch of the convergence proof. Full details will be provided in a forthcoming paper [8].

\section{Presentation of the model}

Let us define the carbonated domain $Q_{s}(T)=\{(y, t): 0<y<s(t), 0<t<T<$ $+\infty\}$. The system considered by Aiki and Muntean in [1] writes

$$
\begin{gathered}
\partial_{t} u-\partial_{y}\left(\kappa_{u} \partial_{y} u\right)=f(u, v) \quad \text { in } \quad Q_{s}(T), \\
\partial_{t} v-\partial_{y}\left(\kappa_{v} \partial_{y} v\right)=-f(u, v) \quad \text { in } Q_{s}(T), \\
s^{\prime}(t)=\psi(u(s(t), t)) \text { for } 0<t<T, \\
s(0)=s_{0}, \\
-\kappa_{u} \partial_{y} u(s(t), t)-s^{\prime}(t) u(s(t), t)=\psi(u(s(t), t)) \quad \text { for } 0<t<T, \\
-\kappa_{v} \partial_{y} v(s(t), t)-s^{\prime}(t) v(s(t), t)=0 \text { for } 0<t<T, \\
u(0, t)=g(t), v(0, t)=r(t) \text { for } 0<t<T, \\
u(y, 0)=u_{0}(y), v(y, 0)=v_{0}(y) \text { for } 0<y<s(0) .
\end{gathered}
$$

Here and hereafter we suppose the following assumptions:

$(A 1) \psi: \mathbb{R} \longrightarrow \mathbb{R}$ drives the carbonation reaction and is defined by $\psi(x)=\alpha x$ with $\alpha>0$,

(A2) $f: \mathbb{R}^{2} \longrightarrow \mathbb{R}$ measures the availability of $\mathrm{CO}_{2}(\mathrm{aq})$ for the carbonation reaction. It is defined by $f(p, q)=\beta(\gamma q-p)$ where $\beta$ and $\gamma$ are two real constants, (A3) $g$ and $r$ belong to $W^{1,2}(0, T)$,

(A4) $u_{0}$ and $v_{0}$ belong to $L^{\infty}\left(\left[0, s_{0}\right]\right)$,

(A5) the diffusive coefficients $\kappa_{u}$ and $\kappa_{v}$ are two positive constants,

(A6) $s_{0}>0$

(A7) there exist $g^{*}$ and $r^{*}$ two positive constants such that $0 \leq g \leq g^{*}, 0 \leq r \leq r^{*}$ on $[0,+\infty)$ and $0 \leq u_{0} \leq g^{*}, 0 \leq v_{0} \leq r^{*}$ on $\left[0, s_{0}\right]$ with $g^{*}=\gamma r^{*}$.

For numerical reasons, it is convenient to rewrite (1) on a fixed space domain. For this, we use the change of variables 


$$
\begin{aligned}
\cup_{0 \leq t \leq T}[0, s(t)] \times\{t\} & \rightarrow[0,1] \times[0, T]=Q(T), \\
(y, t) & \longmapsto\left(x(y, t)=\frac{y}{s(t)}, t\right),
\end{aligned}
$$

and we associate $u(y, t)=\bar{u}(x(y, t), t)$ and $v(y, t)=\bar{v}(x(y, t), t)$. Then if we drop the bars, we obtain, as in [6]

$$
\begin{gathered}
s(t) \partial_{t}(s(t) u)+\partial_{x} J_{u}=s^{2}(t) f(u, v) \text { in } Q(T), \\
s(t) \partial_{t}(s(t) v)+\partial_{x} J_{v}=-s^{2}(t) f(u, v) \text { in } Q(T), \\
s^{\prime}(t)=\psi(u(1, t)) \quad \text { for } 0<t<T, \\
s(0)=s_{0}, \\
J_{u}(1, t)=s(t) \psi(u(1, t)), J_{v}(1, t)=0 \text { for } 0<t<T, \\
u(0, t)=g(t), \quad v(0, t)=r(t) \quad \text { for } 0<t<T, \\
u(x, 0)=u_{0}\left(s_{0} x\right), \quad v(x, 0)=v_{0}\left(s_{0} x\right) \text { for } 0<x<1,
\end{gathered}
$$

with $J_{w}=-\kappa_{w} \partial_{x} w-s(t) s^{\prime}(t) x w$, for $w=u$ or $v$. We now define the notion of weak solution for (2). For this, we introduce the functional space $H=\left\{z \in H^{1}(0,1)\right.$ : $z(0)=0\}$ endowed with the $H^{1}(0,1)$ norm. Assuming $(A 1)-(A 7)$, we say that $(s, u, v)$ is a weak solution of (2) if the following conditions are satisfied

$(S 1)(u, v) \in\left(L^{2}\left(0, T ; H^{1}(0,1)\right) \cap L^{\infty}(Q(T))\right)^{2}$,

(S2) $u-g, v-r \in L^{2}(0, T ; H)$,

(S3) $s \in W^{1, \infty}(0, T), s(0)=s_{0}$ and $s^{\prime}(t)=\psi(u(1, t))$ for almost every $t \in[0, T]$,

(S4) for all $\phi \in \mathscr{C}_{c}^{\infty}([0, T) \times(0,1])$

$$
\begin{aligned}
& -\int_{0}^{T} \int_{0}^{1} u(x, t) s(t) \partial_{t}(s(t) \phi(x, t)) d x d t-\int_{0}^{1} u_{0}\left(s_{0} x\right) s_{0}^{2} \phi(x, 0) d x \\
& +\int_{0}^{T} \int_{0}^{1} \kappa_{u} \partial_{x} u(x, t) \partial_{x} \phi(x, t) d x d t+\int_{0}^{T} \int_{0}^{1} s(t) s^{\prime}(t) x u(x, t) \partial_{x} \phi(x, t) d x d t \\
& +\int_{0}^{T} s(t) \psi(u(1, t)) \phi(1, t) d t=\int_{0}^{T} \int_{0}^{1} s^{2}(t) f(u(x, t), v(x, t)) \phi(x, t) d x d t,
\end{aligned}
$$

(S5) for all $\phi \in \mathscr{C}_{c}^{\infty}([0, T) \times(0,1])$

$$
\begin{aligned}
& -\int_{0}^{T} \int_{0}^{1} v(x, t) s(t) \partial_{t}(s(t) \phi(x, t)) d x d t-\int_{0}^{1} v_{0}\left(s_{0} x\right) s_{0}^{2} \phi(x, 0) d x \\
& +\int_{0}^{T} \int_{0}^{1} \kappa_{v} \partial_{x} v(x, t) \partial_{x} \phi(x, t) d x d t+\int_{0}^{T} \int_{0}^{1} s(t) s^{\prime}(t) x v(x, t) \partial_{x} \phi(x, t) d x d t \\
& =-\int_{0}^{T} \int_{0}^{1} s^{2}(t) f(u(x, t), v(x, t)) \phi(x, t) d x d t .
\end{aligned}
$$




\section{Numerical scheme}

Let us first introduce some notation related to the discretisation of $[0,1]$. A mesh $\mathscr{T}$, consists in a finite sequence of cells $\left(x_{i-\frac{1}{2}}, x_{i+\frac{1}{2}}\right)$ for $1 \leq i \leq l$, with

$$
0=x_{\frac{1}{2}}<x_{\frac{3}{2}}<\ldots<x_{l-\frac{1}{2}}<x_{l+\frac{1}{2}}=1 .
$$

We define $x_{i}$ as the center of the cell $\left(x_{i-\frac{1}{2}}, x_{i+\frac{1}{2}}\right)$ and $x_{0}=x_{\frac{1}{2}}, x_{l+1}=x_{l+\frac{1}{2}}$. We set $h_{i}$ the length of the cell $\left(x_{i-\frac{1}{2}}, x_{i+\frac{1}{2}}\right), h_{i+\frac{1}{2}}$ the length of the interval $\left[x_{i}, x_{i+1}\right]$ and we define $h=\max \left\{h_{i}, 1 \leq i \leq l\right\}$ the size of the mesh. Finally, we define an integer $N_{T}$ and a time step $\Delta t$ such that $N_{T} \Delta t=T$ and $\left(t_{n}\right)_{0 \leq n \leq N_{T}}$ with $t_{n}=n \Delta t$. In the sequel, when we use the notation $w$, it means that $w=u$ or $v$. We discretize the initial and Dirichlet conditions. We set,

$$
\begin{gathered}
s^{0}=s_{0}, \\
w_{i}^{0}=\frac{1}{h_{i}} \int_{x_{i-\frac{1}{2}}}^{x_{i+\frac{1}{2}}} w_{0}\left(s_{0} x\right) d x, \quad \text { for } \quad 1 \leq i \leq l, \text { and } w_{l+1}^{0}=w_{0}\left(s_{0}\right), \\
v_{0}^{n}=\frac{1}{\Delta t} \int_{t_{n}}^{t_{n+1}} r(t) d t, \quad u_{0}^{n}=\frac{1}{\Delta t} \int_{t_{n}}^{t_{n+1}} g(t) d t, \quad \text { for } \quad 0 \leq n \leq N_{T}-1 .
\end{gathered}
$$

Then, for $1 \leq i \leq l$ and $0 \leq n \leq N_{T}-1$, the scheme writes

$$
\begin{gathered}
s^{n+1}=s^{n}+\Delta t \psi\left(u_{l+1}^{n}\right), \\
s^{n+1} h_{i} \frac{s^{n+1} v_{i}^{n+1}-s^{n} v_{i}^{n}}{\Delta t}+\left(G_{v, i+\frac{1}{2}}^{n+1}-G_{v, i-\frac{1}{2}}^{n+1}\right)=-\left(s^{n+1}\right)^{2} h_{i} \beta\left(\gamma v_{i}^{n+1}-u_{i}^{n}\right), \\
s^{n+1} h_{i} \frac{s^{n+1} u_{i}^{n+1}-s^{n} u_{i}^{n}}{\Delta t}+\left(G_{u, i+\frac{1}{2}}^{n+1}-G_{u, i-\frac{1}{2}}^{n+1}\right)=\left(s^{n+1}\right)^{2} h_{i} \beta\left(\gamma v_{i}^{n+1}-u_{i}^{n+1}\right) .
\end{gathered}
$$

It remains to define the numerical fluxes $G_{w, i+\frac{1}{2}}^{n}$. We define $C^{n+1}=s^{n+1}\left(s^{n+1}-\right.$ $\left.s^{n}\right) / \Delta t$ and we use the Scharfetter-Gummel fluxes (see $[7,11]$ ), that is:

$$
G_{w, i+\frac{1}{2}}^{n+1}=\kappa_{w} \frac{B\left(h_{i+\frac{1}{2}} \frac{C^{n+1}}{\kappa_{w}} x_{i+\frac{1}{2}}\right) w_{i}^{n+1}-B\left(-h_{i+\frac{1}{2}} \frac{C^{n+1}}{\kappa_{w}} x_{i+\frac{1}{2}}\right) w_{i+1}^{n+1}}{h_{i+\frac{1}{2}}} .
$$

Here $B$ is the Bernoulli function defined by $B(x)=x /\left(e^{x}-1\right)$ for $x \neq 0$ and $B(0)=1$. We supplement the numerical scheme with Robin/Neumann boundary conditions at $x=1$,

$$
G_{v, l+\frac{1}{2}}^{n+1}=0, \quad G_{u, l+\frac{1}{2}}^{n+1}=s^{n+1} \psi\left(u_{l+1}^{n+1}\right), \quad \text { for } \quad 0 \leq n \leq N_{T}-1 .
$$


We remark that the equations (6), (7) and (8) are decoupled. Thus, if we know $\left(s^{n}, u^{n}, v^{n}\right)$ we can compute $s^{n+1}$ by (6) and then $v^{n+1}$ and $u^{n+1}$ thanks to (7) and (8).

\section{Main results}

Theorem 1. The numerical scheme (3)-(10) admits a unique solution. Moreover, for every $n \geq 0$ and $i \in\{0, \cdots, l+1\}$, we have

$$
0 \leq v_{i}^{n} \leq r^{*}, \quad 0 \leq u_{i}^{n} \leq g^{*}, \quad 0 \leq \frac{s^{n+1}-s^{n}}{\Delta t} \leq \alpha g^{*},
$$

and

$$
0<s^{n+1} \leq s^{0}+T \alpha g^{*}, \quad \text { for } 0 \leq n \leq N_{T} .
$$

Sketch of the proof. We rewrite the scheme (7)-(10) as two independent linear systems. Furthermore, thanks to the boundary condition for $v$ at $x=1$ and the definition of $B$, we can eliminate $v_{l+1}^{n+1}$ as $v_{l+1}^{n+1}=\exp \left(-h_{i+\frac{1}{2}} C^{n+1} / \kappa_{v}\right) v_{l}^{n+1}$, for $n \geq 0$. Hence, as (7)-(8) are decoupled, we obtain two independent linear systems $\mathbb{M}_{v}^{n} v^{n+1}=b_{v}^{n}$ and $\mathbb{M}_{u}^{n} u^{n+1}=b_{u}^{n}$, with, for $n \geq 0, \mathbb{M}_{v}^{n} \in \mathbb{R}^{l \times l}$ and $\mathbb{M}_{u}^{n} \in \mathbb{R}^{(l+1) \times(l+1)}$. Then, following the ideas of [6], we prove that $\mathbb{M}_{v}^{n}$ and $\mathbb{M}_{u}^{n}$ are M-matrices. As a consequence, we deduce the existence of solutions to $(S)$ and the nonnegativity of $u^{n+1}$ and $v^{n+1}$. Finally, we show that $M_{v}^{n}\left(v^{n+1}-r^{*} I_{l}\right) \leq 0$ and $M_{u}^{n}\left(u^{n+1}-g^{*} I_{l+1}\right) \leq 0$ and we deduce the upper bounds of (11) and (12).

In order to use discrete functional analysis tools we introduce some functional sets. Let $\mathscr{T}$ a mesh of $[0,1]$ of size $h$ and $\Delta t$ a time step. We define the set of piecewise constant functions in space as

$$
\begin{aligned}
X_{\mathscr{T}}=\left\{w_{h}:[0,1]\right. & \longrightarrow \mathbb{R}: \exists\left(w_{i}\right)_{0 \leq i \leq l+1} \in \mathbb{R}^{l+2} \text { and } \\
w_{h}(x) & \left.=\sum_{i=1}^{l} w_{i} \mathbf{1}_{\left(x_{i-\frac{1}{2}}, x_{i+\frac{1}{2}}\right)}(x)+w_{0} \mathbf{1}_{\{x=0\}}(x)+w_{l+1} \mathbf{1}_{\{x=1\}}(x)\right\} .
\end{aligned}
$$

We define also the set of piecewise constant functions in space and time as

$$
\begin{aligned}
X_{\mathscr{T}, \Delta t}=\left\{w_{h, \Delta t}:[0,1] \times[0, T] \longrightarrow \mathbb{R}: \exists\left(w_{h}^{k+1}\right)_{0 \leq k \leq N_{T}-1} \in\left(X_{\mathscr{T}}\right)^{N_{T}}\right. \\
\text { and } \left.w_{h, \Delta t}(x, t)=\sum_{k=0}^{N_{T}-1} w_{h}^{k+1}(x) \mathbf{1}_{\left[t_{k}, t_{k+1}\right)}(t)\right\} .
\end{aligned}
$$

Finally, we define a discrete derivative operator in space $\partial_{x, \mathscr{T}}$ for all $w_{h, \Delta t} \in X_{\mathscr{T}, \Delta t}$ by

$$
\partial_{x, \mathscr{T}} w_{h, \Delta t}(x, t)=\partial_{x, \mathscr{T}}^{i} w_{h, \Delta t}=\frac{\left(w_{i+1}^{k+1}-w_{i}^{k}\right)}{h_{i+\frac{1}{2}}} \text { for } \quad(x, t) \in\left(x_{i}, x_{i+1}\right) \times\left(t_{k}, t_{k+1}\right) .
$$


Let $\left(\mathscr{T}_{m}, \Delta t_{m}\right)$ be a sequence of discretizations such that $\left(h_{m}, \Delta t_{m}\right)_{m} \rightarrow 0$. If we consider for each $m$ the approximate solutions $s_{m}, u_{m}$ and $v_{m}$, where $w_{m}=w_{\Delta t_{m}}, h_{m}$ and for $t \in[0, T]$

$$
s_{m}(t)=s_{\Delta t_{m}}(t)=\sum_{k=0}^{N_{T}-1}\left(s^{k+1}+\left(t-t_{k+1}\right) \frac{s^{k+1}-s^{k}}{\Delta t}\right) \mathbf{1}_{\left[t_{k}, t_{k+1}\right)}(t) .
$$

Theorem 2. The sequence $\left(s_{m}, u_{m}, v_{m}\right)_{m}$ converges to $(s, u, v)$ with

$$
\begin{aligned}
w_{m} & \rightarrow w \text { in } L^{2}\left(0, T ; L^{2}(0,1)\right), \\
\partial_{x, T_{m}} w_{m} & \rightarrow \partial_{x} w \text { in } L^{2}\left(0, T ; L^{2}(0,1)\right), \\
s_{m} & \rightarrow s \text { in } \mathscr{C}([0, T]), \\
\partial_{t} s_{m} & \stackrel{w^{*}}{\rightarrow} s^{\prime} \text { in } L^{\infty}(0, T),
\end{aligned}
$$

and $(s, u, v)$ is the weak solution to (2).

Sketch of the proof. First, we establish $L^{2}\left(0, T ; H^{1}(0,1)\right)$ and $H^{1}\left(0, T ; H^{*}\right)$ discrete estimates for $\left(u_{m}\right)_{m}$ and $\left(v_{m}\right)_{m}$, where $H^{*}$ is the dual space to $H$. Then, thanks to a discrete version of the Aubin-Simon lemma [9], we obtain some compactness results for $\left(u_{m}, v_{m}\right)_{m}$ and the convergence of a subsequence towards some limit $(u, v)$. For the sequence $\left(s_{m}\right)_{m}$, we use Theorem 1 and the Ascoli theorem to show that $\left(s_{m}\right)_{m}$ converges to some function $s \in W^{1, \infty}(0, T)$. We also prove the convergence of the traces following [5]. Finally passing to the limit in the numerical scheme as in [4] we obtain that $(s, u, v)$ is the weak solution to (2). As the system (2) admits a unique solution (see [1]), we deduce the convergence of the whole sequence $\left(s_{m}, u_{m}, v_{m}\right)$.

\section{Numerical experiments}

We consider the following test case:

\begin{tabular}{|c|c|c|c|c|c|c|c|c|c|}
\hline$\kappa_{u}$ & $\kappa_{v}$ & $g$ & $r$ & $s_{0}$ & $u_{0}$ & $v_{0}$ & $\alpha$ & $\gamma$ & $\beta$ \\
\hline 1 & 0.1 & 15 & 2.25 & 0.5 & 1 & 1 & 1 & 6.5 & 7.5 \\
\hline
\end{tabular}

Table 1: Definition of the test case.

Fig 1 shows the different profiles of $v$ and $u$ as a function of $x \in[0, s(t)]$ for $t \in$ $\{20,40,60,80,100\}$. We note that the profiles are similar to those given in $[3,10]$. Fig 2 shows the behavior of $s$ for $T=100$ in linear scale and for $T=1000$ in $\log$ arithmic scale. These numerical experiments support the $\sqrt{T}$-law of propagation given in $[3,10]$. 

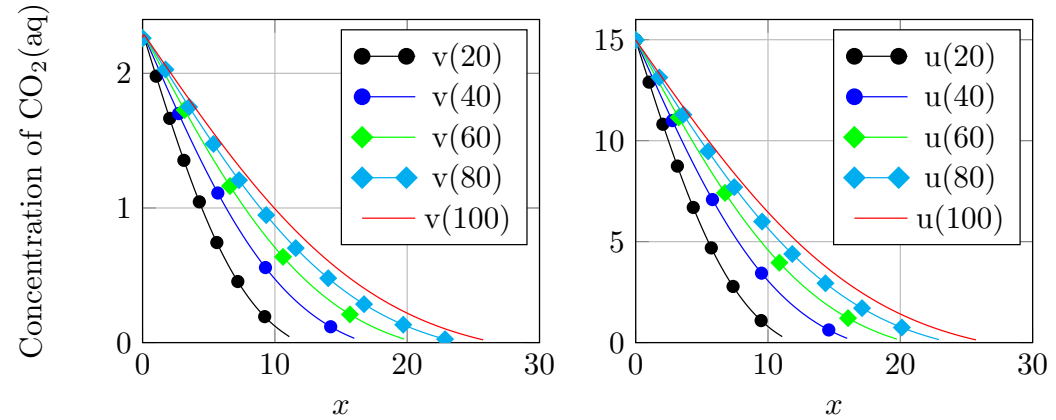

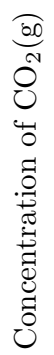

Fig. 1: Profiles of $v$ (on the left) and $u$ at different times. The solutions are plotted on $[0, s(t)]$.
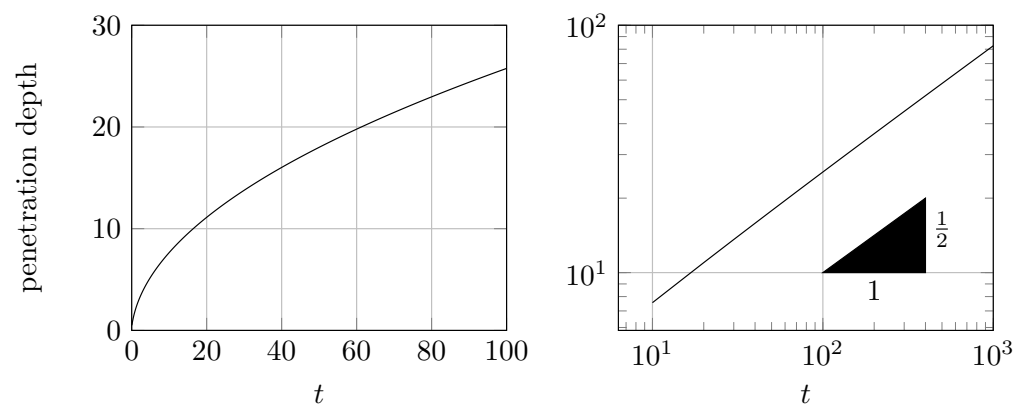

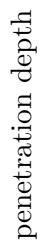

Fig. 2: Behavior of $s$ in linear scale for $T=100$ (on the left) and in logarithmic scale for $T=1000$.

The exact solutions $u$ and $v$ of (2) are not explicitly known. Thus, in order to investigate the question of the $L^{\infty}$ and $L^{2}$-convergence rate in space of the scheme, we compute two reference solutions on a uniform mesh composed of 2560 cells and with $\Delta t=(1 / 2560)^{2}$. Then, we compute the $L^{\infty}$ and $L^{2}$-norm of the difference between the approximate solution and the average of the reference solution over 10 , 20, 40, 80, 160, 320, 640 and 1280 cells. Eventually, in Table 2, we present the $L^{\infty}$ and $L^{2}$-convergence rate in space of the scheme at final time $T=0.1$. As we can observe the scheme has an order around 2 in space, which is consistent with the choice of the Scharfetter-Gummel fluxes.

Acknowledgements The authors are partially supported by the INRIA team RAPSODI, the ANR MOONRISE and the Labex CEMPI (ANR-11-LABX-0007-01). 


\begin{tabular}{|c|c|c|c|c|c|c|c|c|}
\hline \multirow{2}{*}{ cells } & \multicolumn{4}{|c|}{$u$} & \multicolumn{4}{|c|}{$v$} \\
\cline { 2 - 9 } & error $L^{2}$ & order $L^{2}$ & error $L^{\infty}$ & order $L^{\infty}$ & error $L^{2}$ & order $L^{2}$ & error $L^{\infty}$ & order $L^{\infty}$ \\
\hline 10 & $3.5 \mathrm{e}-01$ & - & $4.8 \mathrm{e}-01$ & - & $1.1 \mathrm{e}-01$ & - & $1.6 \mathrm{e}-01$ & - \\
20 & $6.9 \mathrm{e}-02$ & 2.34 & $9.5 \mathrm{e}-02$ & 2.34 & $2.1 \mathrm{e}-02$ & 2.40 & $3.0 \mathrm{e}-02$ & 2.43 \\
40 & $1.6 \mathrm{e}-02$ & 2.09 & $2.2 \mathrm{e}-02$ & 2.08 & $4.9 \mathrm{e}-03$ & 2.10 & $7.0 \mathrm{e}-03$ & 2.10 \\
80 & $4.0 \mathrm{e}-03$ & 2.02 & $5.5 \mathrm{e}-03$ & 2.02 & $1.2 \mathrm{e}-03$ & 2.03 & $1.7 \mathrm{e}-03$ & 2.03 \\
160 & $9.9 \mathrm{e}-04$ & 2.01 & $1.4 \mathrm{e}-03$ & 2.01 & $3.0 \mathrm{e}-04$ & 2.01 & $4.3 \mathrm{e}-04$ & 2.01 \\
320 & $2.5 \mathrm{e}-04$ & 2.02 & $3.4 \mathrm{e}-04$ & 2.02 & $7.4 \mathrm{e}-05$ & 2.02 & $1.1 \mathrm{e}-04$ & 2.02 \\
640 & $5.8 \mathrm{e}-05$ & 2.07 & $8.1 \mathrm{e}-05$ & 2.07 & $1.8 \mathrm{e}-05$ & 2.07 & $2.5 \mathrm{e}-05$ & 2.07 \\
1280 & $1.2 \mathrm{e}-05$ & 2.32 & $1.6 \mathrm{e}-05$ & 2.32 & $3.5 \mathrm{e}-06$ & 2.32 & $5.0 \mathrm{e}-06$ & 2.32 \\
\hline
\end{tabular}

Table 2: $L^{\infty}$ and $L^{2}$-norm of the error for $u$ and $v$ in space.

\section{References}

1. Aiki, T., Muntean, A.: Existence and uniqueness of solutions to a mathematical model predicting service life of concrete structure. Adv. Math. Sci. Appl. 19, 109-129 (2009)

2. Aiki, T., Muntean, A.: Large time behavior of solutions to a moving-interface problem modeling concrete carbonation. Comm. Pure Appl. Anal. 9, 1117-1129 (2010)

3. Aiki, T., Muntean, A.: A free-boundary problem for concrete carbonation: rigorous justification of the $\sqrt{T}$-law of propagation. European Mathematical Society 15, 167-180 (2013)

4. Bessemoulin-Chatard, M.: A finite volume scheme for convection-diffusion equations with nonlinear diffusion derived from the Schafetter-Gummel scheme. Numer. Math. 121(4), 637670 (2013)

5. Brenner, K., Cancès, C., Hilhorst, D.: Finite volume approximation for an immiscible twophase flow in porous media with discontinuous capillary pressure. Comput. Geosci 17(3), 573-597 (2013)

6. Chainais-Hillairet, C., Bataillon, C.: Mathematical and numerical study of a corrosion model. Numer. Math. 110(1), 1-25 (2008)

7. Chainais-Hillairet, C., Droniou, J.: Finite volume schemes for non-coercive elliptic problems with neumann boundary conditions. IMA Journal of Numerical Analysis 31(1), 61-85 (2011)

8. Chainais-Hillairet, C., Merlet, B., Zurek, A.: Convergence of a finite volume scheme for a parabolic system with a free boundary modeling concrete carbonation. hal-01477543 (2017)

9. Gallouët, T., Latché, J.C.: Compactness of discrete approximate solutions to parabolic PDEsapplication to a turbulence model. Commun. Pure Appl. Anal. 11(6), 2371-2391 (2012)

10. Meyer, S., Peter, M., Muntean, A., Böhm, M.: Dynamics of the internal reaction layer arising during carbonation of concrete. Chemical Engineering Sci 62, 1125-1137 (2007)

11. Scharfetter, D., Gummel, H.: Large signal analysis of a silicon read diode oscillator. IEEE Trans. Electron Dev. 16, 64-77 (1969) 